\title{
$\mathrm{PC}$ 鋼線の付加的な影響を考慮した場合のプレス
}

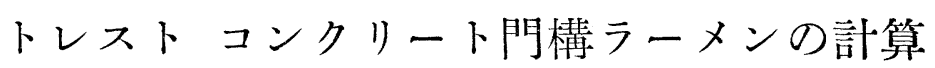

\author{
正員田原保 二* \\ 准員国広哲 男**
}

\section{THE CALCULATION OF PRESTRESSED CONCRETE RIGID FRAME IN CONSIDERATION OF SECONDARY EFFECT OF PRESTRESSING WIRES}

(Trans. of JSCE, No. 29, Dec. 1955)

Yasuji Tahara, C.E. Member and Tetsuo Kunihiro, C. E. Assoc. Member

Synopsis In this paper, the calculation of prestressed concrete rigid frame, in which secondary effect of prestressing wires due to deformations of members is taken into consideration, is shown. Further, the author compares these calculated values with the measured values on Misogi-bashi (Misogi-bridge), the first prestressed concrete bridge of rigid frame type in Japan, and point out this method is rational.

要 旨 本交は部材の変形にともなう PC 鋼線の付加的な影響を考慮に入れた場合のプレストレスト コンクリート門構ラーメンの計算を示したるのであり, さらにこれらの計算值を本邦最初のプレス

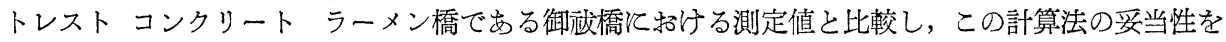
指摘したものである。

\section{1. 緒言}

プレストレストコンクリート構造物が外荷重を受けると部材の変形により PC 鋼線には付加的な緊張力また怯 弛緩力が生じ，そのため 2 次的な付加軸力，曲げモーメントが起る。実際構造物汇現われる軸力，曲げモーメン トは外荷重によるものと PC 鋼線の付加的な影響によつて 2 次的に発生するものとが合成されたものであつて， 後者の影響は不静定構造物では不静定力に関係し無視し得ないると考皇られる。筆者は 29 年七尾市に架設され た本邦最初のプレストレスト コンクリート ラーメン橋である御发橋とついて耐力試験を行つた。以下に耐力試 験に和けるような対称な載荷状態について PC 鋼線の付加的な影響を考慮した場合の計算を示し，実測した応力 並びにタワミと対比して検討を試みた。

図-1

\section{2. 不静定力 $\boldsymbol{H}$ の算出}

載荷状態は図一1のようと対称であり (a), (b) 飞 分けて考觉る。図一2 は以下の計算行用いる断面諸元 の表示である。な持曲げモーメントはハリ部, 脚柱部 とも門構の内側への曲げ, 朝力は圧縮, 付加緊張力は 緊張増加, 長さの変化は縮み, ハリ 端部の回転角は下向きの回転, 脚柱 下端の変位は門構の外側方向への変 位の各場合をそれぞれ正とする。 PC 鋼線の位置は，ハリ部ではハリ の中央 $\mathrm{C}$ 点を $x$ の原点としハリの中 立軸飞対し（1）式により，また脚 柱部では上端を $y$ の原点とし下方に $y>0$ ととり, 脚柱部の中立軸飞対し

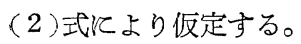
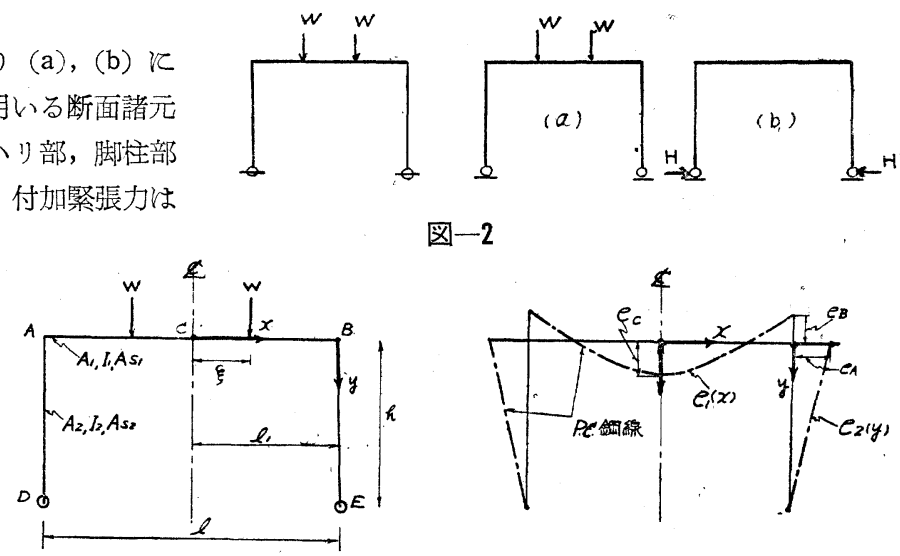

図-2

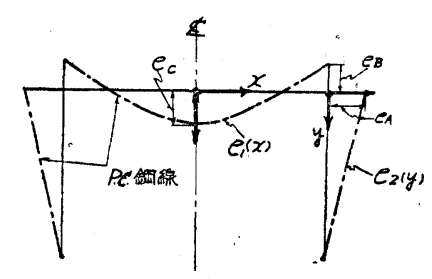

$$
\begin{aligned}
& e_{1}(x)=e_{C}-\frac{e_{B}+e_{C}}{l_{1}{ }^{2}} \cdot x^{2} \text { ただし } l_{1}=\frac{l}{2} \quad e_{B}, e_{C} \geqq 0 \\
& e_{2}(y)=e_{A}(1-y / h) \quad \text { ただし } e_{A} \geqq 0
\end{aligned}
$$

(a) 脚柱下端 $\mathrm{D}, \mathrm{E}$ が自由に水平飞移動しろるとき外力 $W$ 飞よる $\mathrm{D}, \mathrm{E}$ の変位

（i）八リ端部 A,B の回転角とハリ長 $l$ の長さの変化：PC 鋼線の付加緊張力または弛緩力をハリ部，脚柱部

* 建設技官, 建設省土木研究所構造研究室長

** 建設技官, 建設省土木研究所構造研究室 
そついてそれぞれ $\Delta P_{1}(x), \Delta P_{2}(y)$ とすれば，ハリの曲げモーメント $M_{1 W}(x)$ 及び軸力 $N_{1 W}(x)$ は

$$
\begin{aligned}
M_{1 W}(x) & =W\left(l_{1}-\xi\right)-\Delta P_{1}(x) \cdot e_{1}(x)+m_{1 W} & & 0 \leqq x \leqq \xi \\
& =W\left(l_{1}-x\right)-\Delta P_{1}(x) \cdot e_{1}(x)+m_{1 W} & & \xi \leqq x \leqq l_{1}
\end{aligned}
$$

ただし，ここに $m_{1 W}=\Delta P_{2}(0) \cdot e_{2}(0)$

$$
N_{1 W}(x)=\Delta P_{1}(x)
$$

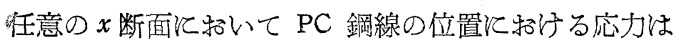

$$
\sigma_{1 W}(x)=\frac{N_{1 W}(x)}{A_{1}}-\frac{M_{1 W}(x)}{I_{1}} \cdot e_{1}(x) \quad 0 \leqq x \leqq l_{1}
$$

中立軸の位置飞和ける応力は

$$
\sigma_{1 W 0}(x)=N_{1 W}(x) / A_{1}
$$

$0 \leqq x \leqq l_{1}$

$\sigma_{1 W}(x)$ そよるコンクリートのヒズミ $\left(\frac{\Delta \delta x}{\delta x}\right)_{W}$ は

$$
\begin{aligned}
\left(\frac{\Delta \delta x}{\delta x}\right)_{W} & =\frac{1}{E_{C}} \sigma_{1 W}(x) \\
& =\frac{1}{E_{C}}\left\{\frac{\Delta P_{1}(x)}{A_{1}}-\frac{W\left(l_{1}-\xi\right)}{I_{1}} e_{1}(x)+\frac{\Delta P_{1}(x)}{I_{1}} e_{1}{ }^{2}(x)-\frac{m_{1 W}}{I_{1}} e_{1}(x)\right\}, 0 \leqq x \leqq \xi \\
& =\frac{1}{E_{C}}\left\{\frac{\Delta P_{1}(x)}{A_{1}}-\frac{W\left(l_{1}-x\right)}{I_{1}} e_{1}(x)+\frac{\Delta P_{1}(x)}{I_{1}} e_{1}{ }^{2}(x)-\frac{m_{1}}{I_{1}} e_{1}(x)\right\}, \quad \xi \leqq x \leqq l_{1}
\end{aligned}
$$

一方

$$
(\Delta \delta x)_{W}=-\frac{1}{E_{S} A_{S 1}} \cdot \Delta P_{1}(x) \cdot \delta x, \quad 0 \leqq x \leqq l_{1}
$$

$$
\begin{aligned}
\therefore \Delta P_{1}(x) & =\frac{W\left(l_{1}-\xi\right)\left(e_{C}-\frac{e x^{2}}{l_{1}{ }^{2}}\right)}{\left(1+\mu_{1}\right) k_{1}{ }^{2}+\left(e_{C}-\frac{e x^{2}}{l_{1}{ }^{2}}\right)^{2}}+\frac{m_{1 W}\left(e_{C}-\frac{e x^{2}}{l_{1}{ }^{2}}\right)}{\left(1+\mu_{1}\right) k_{1}{ }^{2}+\left(e_{C}-\frac{e x^{2}}{l_{1}{ }^{2}}\right)^{2}}, \quad 0 \leqq x \leqq \xi \cdots \\
& =\frac{W\left(l_{1}-x\right)\left(e_{C}-\frac{e x^{2}}{l_{1}{ }^{2}}\right)}{\left(1+\mu_{1}\right) k_{1}{ }^{2}+\left(e_{C}-\frac{e x^{2}}{l_{1}{ }^{2}}\right)^{2}}+\frac{m_{1 W}\left(e_{C}-\frac{e x^{2}}{l_{1}{ }^{2}}\right)}{\left(1+\mu_{1}\right) k_{1}{ }^{2}+\left(e_{C}-\frac{e x^{2}}{l_{1}{ }^{2}}\right)^{2}}, \quad \xi \leqq x \leqq l_{1}
\end{aligned}
$$

ただし $\quad e=e_{B}+e_{C} \geqq 0, \quad k_{1}{ }^{2}=\frac{I_{1}}{A_{1}}>0, \quad \mu_{1}=\frac{E_{C} A_{1}}{E_{S} A_{S 1}}>0$

従つて

$$
\begin{aligned}
(\Delta \delta x)_{W 0} & =\frac{1}{E_{C}} \sigma_{1 W 0}(x) \cdot \delta x \\
& =\frac{W}{E_{C} A_{1}}\left(f_{11}(x) \cdot \delta x+K_{1 W} \cdot f_{10}(x) \cdot \delta x\right), \quad 0 \leqq x \leqq \xi \\
& =\frac{W}{E_{C} A_{1}}\left(f_{12}(x) \cdot \delta x+K_{1 W} \cdot f_{10}(x) \cdot \delta x\right), \quad \xi \leqq x \leqq l_{1} \\
M_{1 W}(x) & =W\left\{\varphi_{11}(x)+K_{1 W} \varphi_{10}(x)\right\}, \quad 0 \leqq x \leqq \xi \ldots \ldots \ldots \ldots \ldots \ldots \ldots \ldots \ldots \ldots \ldots \ldots \ldots \ldots \ldots \ldots \ldots \ldots \ldots \ldots
\end{aligned}
$$

ただし上式中 $K_{1 W}=\frac{m_{1} W}{W} ，$

$f_{11}(x)=\frac{\left(l_{1}-\xi\right)\left(e_{C}-\frac{e x^{2}}{l^{2}}\right)}{\left(1+\mu_{1}\right) k_{1}{ }^{2}+\left(e_{C}-\frac{e x^{2}}{l_{1}{ }^{2}}\right)^{2}}, f_{12}(x)=\frac{\left(l_{1}-x\right)\left(e_{C}-\frac{e x^{2}}{l_{1}{ }^{2}}\right)}{\left(1+\mu_{1}\right) k_{1}{ }^{2}+\left(e_{C}-\frac{e x^{2}}{l_{1}{ }^{2}}\right)^{2}}, f_{10}(x)=\frac{e_{C}-\frac{e x^{2}}{l_{1}{ }^{2}}}{\left(1+\mu_{1}\right) k_{1}{ }^{2}+\left(e_{C}-\frac{e x^{2}}{l_{1}{ }^{2}}\right)^{2}}$
$\varphi_{11}(x)=\frac{\left(1+\mu_{1}\right) k_{1}{ }^{2}\left(l_{1}-\xi\right)}{\left(1+\mu_{1}\right) k_{1}{ }^{2}+\left(e_{C}-\frac{e x^{2}}{l_{1}{ }^{2}}\right)^{2}}, \varphi_{12}(x)=\frac{\left(1+\mu_{1}\right) k_{1}{ }^{2}\left(l_{1}-x\right)}{\left(1-\mu_{1}\right) k_{1}{ }^{2}+\left(e_{C}-\frac{e x^{2}}{l_{1}{ }^{2}}\right)^{2}}, \varphi_{10}(x)=\frac{\left(1+\mu_{1}\right) k_{1}{ }^{2}}{\left(1+\mu_{1}\right) k_{1}{ }^{2}+\left(e_{C}-\frac{e x^{2}}{l_{1}{ }^{2}}\right)^{2}}$

ハリの長さの変化を $l_{1}=\frac{l}{2}$ 対して $\Delta l_{1} W$ とすれば

$$
\Delta l_{1 W}=\int_{0}^{l_{1}}(\Delta \delta x)_{W 0}=\frac{W}{E_{C} A_{1}}\left(\int_{0}^{\xi} f_{11}(x) d x+\int_{\xi}^{l_{1}} f_{12}(x) d x+K_{1 W} \int_{0}^{l_{1}} f_{10}(x) d x\right) .
$$

はたハリ端部 $\mathrm{A}, \mathrm{B}$ の回転角を $\Delta \theta_{1} W$ とすれば 


$$
\Delta \theta_{1 W}=\frac{1}{E_{C} I_{1}} \int_{0}^{l_{1}} M_{1 W}(x) \cdot d x=\frac{W}{E_{C} I_{1}}\left(\int_{0}^{\xi} \varphi_{11}(x) d x+\int_{\xi}^{l_{1}} \varphi_{12}(x) d x+K_{1 W} \int_{0}^{l_{1}} \varphi_{10}(x) d x\right)
$$

(ii) 脚柱下端 $\mathrm{D}, \mathrm{E}$ の変位と柱長 $h$ の長さの変化：脚柱部に生ずる曲げモーメント $M_{2 W}(y)$ 及び軸力 ${ }^{\mathrm{N}} N_{2} W$ (y)

$$
\begin{array}{ll}
M_{2 W}(y)=\Delta P_{2}(y) \cdot e_{2}(y)+m_{2 W}\left(1-\frac{y}{h}\right), & 0 \leqq y \leqq h \\
N_{2 W}(y)=W+\Delta P_{2}(y), & 0 \leqq y \leqq h
\end{array}
$$

ただしここに $m_{2 W}=-\Delta P_{1}\left(l_{1}\right) \cdot e_{1}\left(l_{1}\right)$

任意の $y$ 断面治括いて PC 鋼線の位置に招ける応力は

$$
\sigma_{2 W}(y)=\frac{N_{2 W}(y)}{A_{2}}+\frac{M_{2 W}(y)}{I_{2}} e_{2}(y), \quad 0 \leqq y \leqq h
$$

中立軸の位置飞和ける応力は

$$
\sigma_{2 W_{0}}(y)=\frac{N_{2 W}(y)}{A_{2}}, \quad 0 \leqq y \leqq h
$$

$\sigma_{2 W}(y)$ Kよるコンクリートのヒズミ $\left(\frac{\Delta \delta y}{\delta y}\right)_{W}$ ほ

$$
\begin{aligned}
\left(\frac{\Delta \delta y}{\delta y}\right)_{W} & =\frac{1}{E_{C}} \sigma_{2 W}(y) \\
& =\frac{1}{E_{C}}\left\{\frac{W}{A_{2}}+\frac{\Delta P_{2}(y)}{A_{2}}+\frac{\Delta P_{2}(y)}{I_{2}} e_{2}{ }^{2}(y)+\frac{m_{2 W}\left(1-\frac{y}{h}\right)}{I_{2}} e_{2}(y)\right\}, \quad 0 \leqq y \leqq h
\end{aligned}
$$

一方

$$
(\Delta \delta y)_{W}=-\frac{1}{E_{S} A_{S 2}} \cdot \Delta P_{2}(y) \cdot \delta y
$$

$$
\therefore \Delta P_{2}(y)=-\frac{W_{2}{ }^{2}}{\left(1+\mu_{2}\right) k_{2}{ }^{2}+e_{A}{ }^{2}\left(1-\frac{y}{h}\right)^{2}}-\frac{m_{2 W} e_{A}\left(1-\frac{y}{h}\right)^{2}}{\left(1+\mu_{2}\right) k_{2}{ }^{2}+e_{A}{ }^{2}\left(1-\frac{y}{h}\right)^{2}}, \quad 0 \leqq y \leqq h \text {. }
$$

ただし

$$
\mu_{2}=\frac{E_{C} A_{2}}{E_{S} A_{S_{2}}}>0, \quad k_{2}{ }^{2}=\frac{I_{2}}{A_{2}}>0 \text { とする。 }
$$

従つて

$$
\begin{aligned}
& (\Delta \delta y)_{W_{0}}=\frac{1}{E_{C}} \sigma_{2 W_{0}}(y) \cdot \delta y \\
& =\frac{W}{E_{C} A_{2}}\left\{f_{2}(y) \cdot \delta y+K_{2 W} f_{20}(y) \cdot \delta y\right\}, \quad 0 \leqq y \leqq h \\
& M_{2 W}(y)=W\left\{\varphi_{2}(y)+K_{2 W} \varphi_{20}(y)\right\}, \quad 0 \leqq y \leqq h .
\end{aligned}
$$

ただし上式中 $K_{2 W}=\frac{m_{2 W}}{W}$

$$
\begin{aligned}
& f_{2}(y)=1-\frac{k_{2}{ }^{2}}{\left(1+\mu_{2}\right) k_{2}{ }^{2}+e_{A}{ }^{2}\left(1-\frac{y}{h}\right)^{2}}, f_{20}(y)=-\frac{1}{e_{A}}\left\{1-\frac{\left(1+\mu_{2}\right) k_{2}{ }^{2}}{\left(1+\mu_{2}\right) k_{2}{ }^{2}+e_{A}{ }^{2}\left(1-\frac{y}{h}\right)^{2}}\right\} \\
& \varphi_{2}(y)=-\frac{k_{2}{ }^{2} e_{A}\left(1-\frac{y}{h}\right)}{\left(1+\mu_{2}\right) k_{2}{ }^{2}+e_{A}{ }^{2}\left(1-\frac{y}{h}\right)^{2}}, \quad \varphi_{20}(y)=\frac{1}{e_{A}} \frac{\left(1+\mu_{2}\right) k_{2}{ }^{2} e_{A}\left(1-\frac{y}{h}\right)}{\left(1+\mu_{2}\right) k_{2}{ }^{2}+e_{A}{ }^{2}\left(1-\frac{y}{h}\right)^{2}}
\end{aligned}
$$

柱の長さの変化を $\Delta h_{2 W}$ ，ハリ下端 $\mathrm{D}, \mathrm{E}$ の曲げとよる変位を $\Delta l_{2 W}$ とすれば

$$
\begin{aligned}
& \Delta h_{2 W}=\int_{0}^{h}(\Delta \delta y)_{W 0}=\frac{W}{E_{C} A_{2}}\left(\int_{0}^{h} f_{2}(y) \cdot d y+K_{2 W} \cdot \int_{0}^{h} f_{20}(y) d y\right) . \\
& \Delta l_{2 W}=\frac{1}{E_{C} I_{2}} \int_{0}^{h} \int_{0}^{h} M_{2 W}(y) \cdot d y \cdot d y=\frac{W}{E_{C} I_{2}}\left(\int_{0}^{h} \int_{0}^{h} \varphi_{2}(y) d y \cdot d y\right. \\
& \left.+K_{2 W} \int_{0}^{h} \int_{0}^{h} \varphi_{20}(y) \cdot d y \cdot d y\right\}
\end{aligned}
$$

(iii) $W$ とよる脚柱下端 $\mathrm{D}, \mathrm{E}$ の全変位 $: \Delta_{W}$ そ $W$ る脚柱下端 $\mathrm{D}, \mathrm{E}$ の全変位とすれば 


$$
\begin{aligned}
\Delta_{W} & =\left(h-\Delta h_{2 W}\right) \cdot \Delta \theta_{1 W}-\Delta l_{1 W}+\Delta l_{2 W} \\
& \div h \cdot \Delta \theta_{1 W}-\Delta l_{1 W}+\Delta l_{2 W} \ldots \ldots \ldots \ldots . .
\end{aligned}
$$

(b) 脚柱下端 D,E が自由飞水平飞移動し 3 るとき不静定力 $H$ 飞よる脚柱下端 D,E の変位

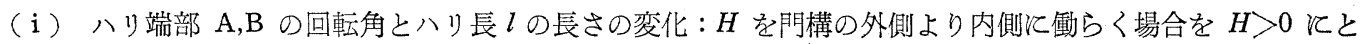
(i), PC 鋼線の緊張力の変化をハリ部, 脚柱部についてそれぞれ $\Delta Q_{1}(x), \Delta Q_{2}(y)$ とすれね゙八りそ動く曲げモー メント $M_{1 H}(x)$ 及び軸力 $N_{1 H}(x)$ は

$$
\begin{array}{ll}
M_{1 H}(x)=-H h-\Delta Q_{1}(x) \cdot e_{1}(x)+m_{1 H}, & 0 \leqq x \leqq l_{1} \\
N_{1 H}(x)=H+\Delta Q_{1}(x), & 0 \leqq x \leqq l_{1}
\end{array}
$$

ただしここと $m_{1 H}=\Delta Q_{2}(0) \cdot e_{2}(0)$

(a) と同様㲸して $\Delta Q_{1}(x)$ を計算すれば

従つて

$$
\Delta Q_{1}(x)=-\frac{H\left\{k_{1}{ }^{2}+h\left(e_{C}-\frac{e x^{2}}{l_{1}{ }^{2}}\right)\right\}}{\left(1+\mu_{1}\right) k_{1}{ }^{2}+\left(e_{C}-\frac{e x^{2}}{l_{1}{ }^{2}}\right)^{2}}+\frac{m_{1 H}\left(e_{C}-\frac{e x^{2}}{l_{1}{ }^{2}}\right)}{\left(1+\mu_{1}\right) k_{1}{ }^{2}+\left(e_{C}-\frac{e x^{2}}{l_{1}{ }^{2}}\right)^{2}}, \quad 0 \leqq x \leqq l_{1} \cdots
$$

$$
\begin{aligned}
& (\Delta \delta x)_{H_{0}}=\frac{H}{E_{C} A_{1}}\left(F_{1}(x) \cdot \delta x+K_{1 H} \cdot F_{10}(x) \cdot \delta x\right), \quad 0 \leqq x \leqq l_{1} \ldots \\
& M_{1 H}(x)=H\left\{\Phi_{1}(x)+K_{1 H} \Phi_{10}(x)\right\}, \quad 0 \leqq x \leqq l_{1} \ldots \ldots \ldots \ldots \ldots \ldots \ldots \ldots \ldots \ldots \ldots \ldots \ldots \ldots
\end{aligned}
$$

ただし上式中 $K_{1 H}=\frac{m_{1 H}}{H}$

$$
\begin{aligned}
& F_{1}(x)=1-\frac{k_{1}{ }^{2}+h\left(e_{C}-\frac{e x^{2}}{l_{1}{ }^{2}}\right)}{\left(1+\mu_{1}\right) k_{1}{ }^{2}+\left(e_{C}-\frac{e x^{2}}{l_{1}{ }^{2}}\right)^{2}}, F_{10}(x)=\frac{e_{C}-\frac{e x^{2}}{l_{1}{ }^{2}}}{\left(1+\mu_{1}\right) k_{1}{ }^{2}+\left(e_{C}-\frac{e x^{2}}{l_{1}{ }^{2}}\right)^{2}} \\
& \Phi_{1}(x)=-\frac{k_{1}{ }^{2}\left\{\left(1+\mu_{1}\right) h-\left(e_{C}-\frac{e x^{2}}{l_{1}{ }^{2}}\right)\right\}}{\left(1+\mu_{1}\right) k_{1}{ }^{2}+\left(e_{C}-\frac{e x^{2}}{l_{1}{ }^{2}}\right)^{2}}, \Phi_{10}(x)=\frac{\left(1+\mu_{1}\right) k_{1}{ }^{2}}{\left(1+\mu_{1}\right) k_{1}{ }^{2}+\left(e_{C}-\frac{e x^{2}}{l_{1}{ }^{2}}\right)^{2}}
\end{aligned}
$$

ハリの長さの変化を $\Delta l_{1 H}$, ハリ端 $\mathrm{A}, \mathrm{B}$ の回転角を $\Delta \theta_{1 H}$ とすれば

$$
\begin{aligned}
& \Delta l_{1 H}=\int_{0}^{l_{1}}(\Delta \delta x)_{H 0}=\frac{H}{E_{C} A_{1}}\left(\int_{0}^{l_{1}} F_{1}(x) \cdot d x+K_{1 H} \int_{0}^{l_{1}} F_{10}(x) d x\right) \ldots \ldots \ldots \ldots . . . . . . . \\
& \Delta \theta_{1 H}=\frac{1}{E_{C} I_{1}} \int_{0}^{l_{1}} M_{1 H}(x) d x=\frac{H}{E_{C} I_{1}}\left(\int_{0}^{l_{1}} \Phi_{1}(x) d x+K_{1 H} \int_{0}^{l_{1}} \Phi_{10}(x) \cdot d x\right)
\end{aligned}
$$

(ii). 脚柱下端 D,E の変位と柱長 $h$ の長さの変化: 脚柱部泟生ずる曲げモーメント $M_{2 H}(y)$ 及び軸力 $N_{2 H}$ (y) ほ

$$
\begin{array}{ll}
M_{2 H}(y)=-H(h-y)+\Delta Q_{2}(y) \cdot e_{2}(y)+m_{2 H}\left(1-\frac{y}{h}\right), & 0 \leqq y \leqq h \\
N_{2 H}(y)=\Delta Q_{2}(y) & 0 \leqq y \leqq h
\end{array}
$$

ただし,ここマ $m_{2 H}=-\Delta Q_{1}\left(l_{1}\right) \cdot e_{1}\left(l_{1}\right)$

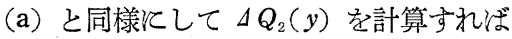

従つて

$$
\Delta Q_{2}(y)=\frac{H h e_{A}\left(1-\frac{y}{h}\right)^{2}}{\left(1+\mu_{2}\right) k_{2}{ }^{2}+e_{A}{ }^{2}\left(1-\frac{y}{h}\right)^{2}}-\frac{m_{2 H e_{A}\left(1-\frac{y}{h}\right)^{2}}}{\left(1+\mu_{2}\right) k_{2}{ }^{2}+e_{A}{ }^{2}\left(1-\frac{y}{h}\right)^{2}}, 0 \leqq y \leqq h
$$

$$
\begin{aligned}
& (\Delta \delta y)_{H 0}=\frac{H}{E_{C} A_{2}}\left\{F_{2}(y) \cdot \delta y+K_{2 H} \cdot F_{20}(y) \cdot \delta y\right\}, \quad 0 \leqq y \leqq h . \\
& \left.M_{2 H}(y)=H\left\{\Phi_{2}(y)+K_{2 H} \Phi_{20}(y)\right)\right\}, \quad 0 \leqq y \leqq h \ldots \ldots \ldots \ldots \ldots \ldots \ldots \ldots \ldots \ldots \ldots \ldots \ldots
\end{aligned}
$$

ただし上式中 $K_{2 H}=\frac{m_{2 H}}{H}$

$$
F_{2}(y)=\frac{h}{e_{A}}\left\{1-\frac{\left(1+\mu_{2}\right) k_{2}{ }^{2}}{\left(1+\mu_{2}\right) k_{2}{ }^{2}+e_{A}{ }^{2}\left(1-\frac{y}{h}\right)^{2}}\right\}, F_{20}(y)=-\frac{1}{e_{A}}\left\{1-\frac{\left(1+\mu_{2}\right) k_{2}{ }^{2}}{\left(1+\mu_{2}\right) k_{2}{ }^{2}+e_{A}{ }^{2}\left(1-\frac{y}{h}\right)^{2}}\right\}
$$




$$
\Phi_{2}(y)=-\frac{h}{e_{A}} \cdot \frac{\left(1+\mu_{2}\right) k_{2}{ }^{2} \cdot e_{A}\left(1-\frac{y}{h}\right)}{\left(1+\mu_{2}\right) k_{2}{ }^{2}+e_{A}{ }^{2}\left(1-\frac{y}{h}\right)^{2}}, \quad \Phi_{20}(y)=\frac{1}{e_{A}} \cdot \frac{\left(1+\mu_{2}\right) k_{2}{ }^{2} e_{A}\left(1-\frac{y}{h}\right)}{\left(1+\mu_{2}\right) k_{2}{ }^{2}+e_{A}{ }^{2}\left(1-\frac{y}{h}\right)^{2}}
$$

柱の長さの変化 $\Delta h_{2 H}$ 及び脚柱下端 $\mathrm{D}, \mathrm{E}$ の曲げとよる変位 $\Delta l_{2 H}$ は

$$
\begin{aligned}
& \Delta h_{2 H}=\int_{0}^{h}(\Delta \delta y)_{H 0}=\frac{H}{E_{C} A_{2}}\left(\int_{0}^{h} F_{2}(y) d y+K_{2 H} \int_{0}^{h} F_{20}(y) d y\right) \\
& \Delta l_{2 H}=\frac{1}{E_{C} I_{2}} \int_{0}^{h} \int_{0}^{h}{ }^{h} M_{2 H}(y) d y \cdot d y=\frac{H}{E_{C} I_{2}}\left(\int_{0}^{h} \int_{0}^{h} \Phi_{2}(y) d y \cdot d y\right. \\
& \left.+K_{2 H} \int_{0}^{h} \int_{0}^{h} \Phi_{20}(y) d y \cdot d y\right)
\end{aligned}
$$

(iii) $H$ そよる脚柱下端 D,E の全変位 $: \Delta_{H}$ を $H$ とよる脚柱下端 $\mathrm{D}, \mathrm{E}$ の全変位とすれば

$$
\begin{aligned}
& \Delta_{H}=\left(h-\Delta h_{2 H}\right) \cdot \Delta \theta_{1 H}-\Delta l_{1 H}+\Delta l_{2 H} \\
& \doteqdot h \cdot \Delta \theta_{1 H}-\Delta l_{1 H}+\Delta l_{2 H}
\end{aligned}
$$

(c) $K_{1 W}, K_{2 W}, K_{1 H}, K_{2 H}$ の算出: 式 $\left(3^{\prime}\right),(8)$ より

$$
\begin{aligned}
& \Delta P_{1}\left(l_{1}\right)=-\frac{e_{A} \cdot e_{B}}{\left(1+\mu_{1}\right) k_{1}{ }^{2}+e_{B}{ }^{2}} \cdot \Delta P_{2}(0) \therefore \\
& \Delta P_{2}(0)=-\frac{W k_{2}{ }^{2}}{\left(1+\mu_{2}\right) k_{2}{ }^{2}+e_{A}{ }^{2}}-\frac{e_{A} \cdot e_{B}}{\left(1+\mu_{2}\right) k_{2}{ }^{2}+e_{A}{ }^{2}} \cdot \Delta P_{1}\left(l_{1}\right)
\end{aligned}
$$

$\left(1+\mu_{1}\right) k_{1}{ }^{2}=\alpha_{1}{ }^{2},\left(1+\mu_{2}\right) k_{2}{ }^{2}=\alpha_{2}{ }^{2}$ と扔いてこの両式を連立的解けげ

式 (14)，(19)より

$$
\Delta P_{1}\left(l_{1}\right)=\frac{k_{2}^{2} e_{A} e_{B}}{\alpha_{1}^{2} \alpha_{2}^{2}+\alpha_{1}^{2} e_{A}{ }^{2}+\alpha_{2}^{2} e_{B}^{2}} \cdot W, \quad \Delta P_{2}(0)=-\frac{k_{2}{ }^{2}\left(\alpha_{1}{ }^{2}+e_{B}{ }^{2}\right)}{\alpha_{1}^{2} \alpha_{2}^{2}+\alpha_{1}^{2} e_{A}{ }^{2}+\alpha_{2}^{2} e_{B}{ }^{2}} \cdot W
$$

$$
\begin{aligned}
& \Delta Q_{1}\left(l_{1}\right)=-\frac{H\left(k_{1}^{2}-h e_{B}\right)}{\left(1+\mu_{1}\right) k_{1}^{2}+e_{B}{ }^{2}}-\frac{e_{A} \cdot e_{B}}{\left(1+\mu_{1}\right) k_{1}{ }^{2}+e_{B}{ }^{2}} \cdot \Delta Q_{2}(0) \\
& \Delta Q_{2}(0)=\frac{H h e_{A}}{\left(1+\mu_{2}\right) k_{2}{ }^{2}+e_{A}{ }^{2}}-\frac{e_{A} \cdot e_{B}}{\left(1+\mu_{2}\right) k_{2}{ }^{2}+e_{A}{ }^{2}} \cdot \Delta Q_{1}\left(l_{1}\right) \cdots
\end{aligned}
$$

この両式を連立的に解けぼ

$$
\Delta Q_{1}\left(l_{1}\right)=-\frac{k_{1}{ }^{2} \alpha_{2}{ }^{2}+k_{1}{ }^{2} e_{A}{ }^{2}-h \alpha_{2}{ }^{2} e_{B}}{\alpha_{1}{ }^{2} \alpha_{2}{ }^{2}+\alpha_{1}{ }^{2} e_{A}{ }^{2}+\alpha_{2}{ }^{2} e_{B}{ }^{2}} H, \quad \Delta Q_{2}(0)=\frac{h \alpha_{1}{ }^{2} e_{A}+e_{A} e_{B} k_{1}{ }^{2}}{\alpha_{1}^{2} \alpha_{2}{ }^{2}+\dot{\alpha}_{1}{ }^{2} e_{A}{ }^{2}+\alpha_{2}{ }^{2} e_{B}{ }^{2}} H
$$

。従つて

$$
\begin{aligned}
& K_{1 W}=\frac{\Delta P_{2}(0) \cdot e_{2}(0)}{W}=-\frac{k_{2}{ }^{2}\left(\alpha_{1}{ }^{2}+e_{B}{ }^{2}\right) e_{A}}{\alpha_{1}{ }^{2} \alpha_{2}{ }^{2}+\alpha_{1}{ }^{2} e_{A}{ }^{2}+\alpha_{2}{ }^{2} e_{B}{ }^{2}} \\
& K_{2 W}=-\frac{\Delta P_{1}\left(l_{1}\right) \cdot e_{1}\left(l_{1}\right)}{W}=\frac{k_{2}{ }^{2} e_{A} e_{B}{ }^{2}}{\alpha_{1}{ }^{2} \alpha_{2}{ }^{2}+\alpha_{1}^{2} e_{A}{ }^{2}+\alpha_{2}{ }^{2} e_{B}{ }^{2}} \\
& K_{1 H}=\frac{\Delta Q_{2}(0) \cdot e_{2}(0)}{H}=\frac{\left(h \alpha_{1}{ }^{2} e_{A}+k_{1}{ }^{2} e_{A} e_{B}\right) e_{A}}{\alpha_{1}^{2} \alpha_{2}{ }^{2}+\alpha_{2}{ }^{2} e_{B}{ }^{2}+\alpha_{1}^{2} e_{A}{ }^{2}} \\
& K_{2 H}=-\frac{\Delta Q_{1}\left(l_{1}\right) e_{1}\left(l_{1}\right)}{H}=-\frac{\left(k_{1}^{2} \alpha_{2}{ }^{2}+k_{1}{ }^{2} e_{A}{ }^{2}-h \alpha_{2}{ }^{2} e_{B}\right) e_{B}}{\alpha_{1}{ }^{2} \alpha_{2}{ }^{2}+\alpha_{2}{ }^{2} e_{B}{ }^{2}+\alpha_{1}^{2} e_{A}{ }^{2}}
\end{aligned}
$$

(d) 不静定力 $H$ の決定: $\Delta_{W}+\Delta_{H}=0$ なるべきとより

$$
\begin{aligned}
& h \cdot \Delta \theta_{1 W}-\Delta l_{1 W}+\Delta l_{2 W}+h \cdot \Delta \theta_{1 H}-\Delta l_{1 H}+\Delta l_{2 H}=0 \\
& \therefore \quad H=-W \cdot \frac{\frac{h}{k_{1}{ }^{2}}\left(J_{11}+J_{12}+K_{1 W} J_{10}\right)-\left(J_{21}+J_{22}+K_{1 W} J_{20}\right)+\frac{\nu}{k_{2}{ }^{2}}\left(J_{3}+K_{2 W} J_{30}\right)}{\frac{h}{k_{1}{ }^{2}}\left(J_{1}{ }^{\prime}+K_{1 H} J_{10}{ }^{\prime}\right)-\left(J_{2}{ }^{\prime}+K_{1 H} J_{20}{ }^{\prime}\right)+\frac{\nu}{k_{2}{ }^{2}}\left(J_{3}{ }^{\prime}+K_{2 H} J_{30}{ }^{\prime}\right)}
\end{aligned}
$$

ただし上式中

$$
\begin{aligned}
& \nu=\frac{A_{1}}{A_{2}}, J_{11}=\int_{0}^{\xi} \varphi_{11}(x) d x, \quad J_{12}=\int_{\xi}^{l_{1}} \varphi_{12}(x) d x, \quad J_{10}=\int_{0}^{l_{1}} \varphi_{10}(x) d x \\
& J_{21}=\int_{0}^{\xi} f_{11}(x) d x, \quad J_{22}=\int_{\xi}^{l_{1}} f_{12}(x) d x, \quad J_{20}=\int_{0}^{l_{1}} f_{10}(x) d x, \quad J_{3}=\int_{0}^{h} \int_{0}^{h} \varphi_{2}(y) d y \cdot d y \\
& J_{30}=\int_{0}^{h} \int_{0}^{h} \varphi_{20}(y) d y \cdot d y, \quad J_{1}{ }^{\prime}=\int_{0}^{l_{1}} \Phi_{1}(x) d x, \quad J_{10}{ }^{\prime}=\int_{0}^{l_{1}} \Phi_{10}(x) d x, \quad J_{2}{ }^{\prime}=\int_{0}^{l_{1}} F_{1}(x) d x
\end{aligned}
$$




$$
J_{20}{ }^{\prime}=\int_{0}^{l_{1}} F_{10}(x) d x, \quad J_{3}{ }^{\prime}=\int_{0}^{h} \int_{0}^{h} \Phi_{2}(y) d y \cdot d y, \quad J_{30}{ }^{\prime}=\int_{0}^{h} \int_{0}^{h} \Phi_{20}(y) d y \cdot d y
$$

これらを計算すると

$$
\begin{aligned}
J_{11}+J_{12}+K_{1 W} J_{10}= & \frac{\alpha_{1}{ }^{2}}{4 K_{1}}\left[\frac{1}{\omega}\left\{l_{1} L_{1}\left(l_{1}\right)+K_{1} L_{1}\left(l_{1}\right)-\xi L_{1}(\xi)\right\}\right. \\
& \left.+\frac{1}{\beta_{1} \gamma}\left\{l_{1} \Theta_{1}\left(l_{1}\right)+K_{1 W} \Theta_{1}\left(l_{1}\right)-\xi \Theta_{1}(\xi)\right\}\right]+\frac{\alpha_{1}}{2 \beta_{1}}\left\{\Theta_{2}\left(l_{1}\right)-\Theta_{2}(\xi)\right\} \\
J_{21}+J_{22}+K_{1 W} J_{20}= & \frac{1}{4}\left[\frac{K_{2}}{\omega}\left\{l_{1} L_{1}\left(l_{1}\right)+K_{1 W} L_{1}\left(l_{1}\right)-\xi L_{1}(\xi)\right\}\right. \\
& \left.-\frac{K_{3}}{\beta_{1} \gamma}\left\{l_{1} \Theta_{1}\left(l_{1}\right)+K_{1 W} \Theta_{1}\left(l_{1}\right)-\xi \Theta_{1}(\xi)\right\}+\frac{1}{\beta_{1}}\left\{L_{2}\left(l_{1}\right)-L_{2}(\xi)\right\}\right] \\
J_{3}+K_{2 W} J_{30}= & \frac{1}{\beta_{2}{ }^{2}}\left(K_{2}{ }^{2} \frac{\alpha_{2}{ }^{2}}{e_{A}}-k_{2}{ }^{2}\right)\left(e_{A}-\alpha_{2} \tan ^{-1} \frac{e_{A}}{\alpha_{2}}\right) \\
J_{1}{ }^{\prime}+K_{1 H} J_{10}{ }^{\prime}= & -\frac{\alpha_{1}{ }^{2}}{4 K_{1}}\left\{\frac{1}{\omega} L_{1}\left(l_{1}\right)+\frac{1}{\beta_{1} \gamma} \Theta_{1}\left(l_{1}\right)\right\}\left(h-K_{1 H}\right)+\frac{k_{1}{ }^{2}}{4}\left\{\frac{K_{2}}{\omega} L_{1}\left(l_{1}\right)-\frac{\left.K_{3} \cdot \Theta_{1}\left(l_{1}\right)\right\}}{\beta_{1} \gamma}\right\} \\
J_{2}{ }^{\prime}+K_{1 H} J^{\prime}{ }_{20}= & l_{1}-\frac{k_{1}{ }^{2}}{4 K_{1}}\left\{\frac{1}{\omega} \cdot L_{1}\left(l_{1}\right)+\frac{1}{\beta_{1} \gamma} \Theta_{1}\left(l_{1}\right)\right\}-\frac{1}{4}\left\{\frac{K_{2}}{\omega} L_{1}\left(l_{1}\right)-\frac{K_{3}}{\beta_{1} \gamma} \Theta_{1}\left(l_{1}\right)\right\}\left(h-K_{1 H}\right) \\
J_{3}{ }^{\prime}+K_{2 H} J_{30}{ }^{\prime}= & \frac{\alpha_{2}{ }^{2}}{\beta_{2}{ }^{2}}\left(\frac{K_{2 H}}{e_{A}}-\frac{1}{\beta_{2}}\right)\left(e_{A}-\alpha_{2} \tan ^{-1} \frac{e_{A}}{\alpha_{2}}\right)
\end{aligned}
$$

ただしここに

$$
\begin{aligned}
& \beta_{1}=\frac{e}{l_{1}{ }^{2}}, \quad \gamma^{2}=\frac{\sqrt{e_{C}{ }^{2}+\alpha_{1}{ }^{2}}-e_{C}}{2 \beta_{1}}, \quad \omega^{2}=2 \beta_{1}\left(\sqrt{e_{C}{ }^{2}+\alpha_{1}{ }^{2}}+e_{C}\right), \\
& \beta_{2}=\frac{e_{A}}{h}, \quad K_{1}=\sqrt{e_{C}{ }^{2}+\alpha_{1}^{2}}, \quad K_{2}=1+\frac{e_{C}}{\sqrt{e_{C}{ }^{2}+\alpha_{1}^{2}}}, \quad K_{3}=1-\frac{e_{C}}{\sqrt{e_{C}{ }^{2}+\alpha_{1}^{2}}} \\
& L_{1}(x)=\log \frac{\beta_{1} x^{2}+\omega x+\sqrt{\beta_{1}{ }^{2}+\alpha_{1}^{2}}}{\beta_{1} x^{2}-\omega x+\sqrt{e_{C}{ }^{2}+\alpha_{1}{ }^{2}}}, \quad L_{2}(x)=\log \left\{\alpha_{1}^{2}+\left(e_{C}-\beta_{1} x^{2}\right)^{2}\right\} \\
& \Theta_{1}(x)=\tan ^{-1} \frac{x-\frac{\omega}{2 \beta_{1}}}{r}+\tan ^{-1} \frac{x+\frac{\omega}{2 \beta_{1}}}{r}, \quad \Theta_{2}(x)=\tan ^{-1} \frac{e_{C}-\beta_{1} x^{2}}{\alpha_{1}}
\end{aligned}
$$

\section{3. 曲げモーメントおよび軸力}

ハリ部及び脚柱部に括ける曲げモーメントを $M_{1}(x), M_{2}(y)$ とすれば

$$
\begin{aligned}
M_{1}(x) & =M_{1 W}(x)+M_{1 H}(x) \\
& =W\left\{\varphi_{11}(x)+K_{1 W} \varphi_{10}(x)\right\}+H\left\{\Phi_{1}(x)+K_{1 H} \Phi_{10}(x)\right\}, \quad 0 \leqq x \leqq \xi \\
& =W\left\{\varphi_{12}(x)+K_{1 W} \varphi_{10}(x)\right\}+\left\{\Phi_{1}(x)+K_{1 H} \Phi_{10}(x)\right\}, \quad \xi \leqq x \leqq l_{1} \cdots \\
M_{2}(y) & =M_{2 W}(y)+M_{2 H}(y) \\
& =W\left\{\varphi_{2}(y)+K_{2 W} \varphi_{20}(y)\right\}+H\left\{\Phi_{2}(y)+K_{2 H} \Phi_{20}(y)\right\}, \quad 0 \leqq y \leqq h \ldots
\end{aligned}
$$

また軸力をハリ部，脚柱部とついてそれぞれ $N_{1}(x), N_{2}(y)$ とすれば

$$
\begin{aligned}
N_{1}(x) & =N_{1 W}(x)+N_{1 H}(x) & & \\
& =W\left\{f_{11}(x)+K_{1 W} f_{10}(x)\right\}+H\left\{F_{1}(x)+K_{1 H} F_{10}(x)\right\}, & & 0 \leqq x \leqq \xi \\
& =W\left\{f_{12}(x)+K_{1 W} f_{10}(x)\right\}+H\left\{F_{1}(x)+K_{1 H} F_{10}(x)\right\}, & & \xi \leqq x \leqq l_{1} \\
N_{2}(y) & =N_{2 W}(y)+N_{2 H}(y) & & \\
& =W\left\{f_{2}(y)+K_{2 W} f_{20}(y)\right\}+H\left\{F_{2}(y)+K_{2 H} F_{20}(y)\right\}, & & 0 \leqq y \leqq h \cdots
\end{aligned}
$$

\section{4. 八リ部におけるタワミ曲線}

図-3

図一3 のごとくハリの中央 $\mathrm{C}$ 点を座標の原点にとり $\eta(x)$ は上向きを正とする。

$$
\frac{d^{2} \eta(x)}{d x^{2}}=\frac{M_{1}(x)}{E_{C} I_{1}}
$$

従つてタワミ $\eta(x)$ は次のよう飞求まる。

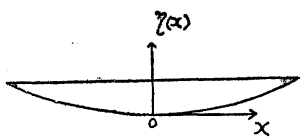




$$
\begin{aligned}
\eta(x) & =\frac{1}{E_{C} I_{1}} \iint M_{1}(x) d x \cdot d x \\
& =\frac{W}{E_{C} I_{1}}\left\{R_{11}(x)+K_{1 W} R_{10}(x)\right\}+\frac{H}{E_{C} I_{1}}\left\{R_{1}^{\prime}(x)+K_{1 H} R_{10}{ }^{\prime}(x)\right\}, \quad 0 \leqq x \leqq \xi \\
& =\frac{W}{E_{C} I_{1}}\left\{R_{12}(x)+K_{1 W} R_{10}(x)\right\}+\frac{H}{E_{C} I_{1}}\left\{R_{1}^{\prime}(x)+K_{1 H} R_{10}{ }^{\prime}(x)\right\}, \quad \xi \leqq x \leqq l_{1} \cdots
\end{aligned}
$$

境界条件を満たすように積分常数を定め， $R_{11}(x), R_{12}(x), R_{10}(x), R_{1}^{\prime}(x), R_{10}{ }^{\prime}(x)$ を計算すると

$$
\begin{aligned}
R_{11}(x) & =\iint \varphi_{11}(x) d x \cdot d x=\frac{1}{4} C_{1}\left(l_{1}-\xi\right)\left\{\frac{x}{\omega} L_{1}(x)+\frac{x}{\beta_{1} \gamma} \cdot \Theta_{1}(x)+C_{2}\left\{\Theta_{3}(x)-\Theta_{3}(0)\right\}\right\} \\
R_{12}(x) & =\iint \varphi_{12}(x) \cdot d x \cdot d x=\frac{1}{4}\left[C_{1} \cdot \frac{x}{\omega}\left\{l_{1} L_{1}(x)-\xi L_{1}(\xi)\right\}+C_{1} \frac{x}{\beta_{1} \gamma}\left\{l_{1} \Theta_{1}(x)-\xi \Theta_{1}(\xi)\right\}\right. \\
& -\frac{C_{3}}{\omega}\left\{L_{1}(x)-L_{1}(\xi)\right\}+\frac{C_{3}}{\beta_{1} \gamma}\left\{\Theta_{1}(x)-\Theta_{1}(\xi)\right\}+C_{4} x\left\{\Theta_{2}(x)-\Theta_{2}(\xi)\right\} \\
& \left.+C_{4}\left\{l_{1} \Theta_{3}(x)-\xi \Theta_{3}(\xi)-\left(l_{1}-\xi\right) \Theta_{3}(0)\right\}\right] \\
R_{10}(x) & =\iint \varphi_{10}(x) d x \cdot d x=\frac{1}{4} C_{1}\left\{\frac{x}{\omega} L_{1}(x)+\frac{x}{\beta_{1} \gamma} \Theta_{1}(x)+C_{2}\left\{\Theta_{3}(x)-\Theta_{3}(0)\right\}\right\} \\
R_{1}^{\prime}(x) & =\iint \Phi_{1}(x) d x \cdot d x=\frac{1}{4}\left\{\frac{C_{5}}{\omega} \cdot x \cdot L_{1}(x)-\frac{C_{6}}{\beta_{1} \gamma} \cdot x \cdot \Theta_{1}(x)\right. \\
& \left.\left.\left.+C_{7}\left\{L_{3}(x)-L_{3}(0)\right\}+C_{8}\right\} \Theta_{3}(x)-\Theta_{3}(0)\right\}\right\} \\
R_{10}{ }^{\prime}(x) & =\iint \Phi_{10}(x) d x d x=R_{10}(x)
\end{aligned}
$$

ただし上式中

$$
\begin{aligned}
& C_{1}=\frac{\alpha_{1}{ }^{2}}{K_{1}}, \quad C_{2}=\frac{2 K_{1}}{\beta_{1} \alpha_{1}}, \quad C_{3}=\frac{\alpha_{1}{ }^{2}}{\beta_{1}}, \quad C_{4}=\frac{2 \alpha_{1}}{\beta_{1}}, \quad C_{5}=k_{1}{ }^{2} K_{2}-\frac{\alpha_{1}{ }^{2} h}{K_{1}} \\
& C_{6}=k_{1}{ }^{2} K_{3}+\frac{\alpha_{1}{ }^{2} h}{K_{1}}, \quad C_{7}=\frac{k_{1}{ }^{2}}{\beta_{1}}, \quad C_{8}=-\frac{2 \alpha_{1}{ }^{2} h}{\beta_{1} \gamma \omega} \\
& \Theta_{3}(x)=\tan ^{-1} \frac{x+\frac{\omega}{2 \beta_{1}}}{\gamma}-\tan ^{-1} \frac{x-\frac{\omega}{2 \beta_{1}}}{r} \\
& L_{3}(x)=\log \left\{\frac{\left(\beta_{1} x^{2}+\omega x+\sqrt{\left.e_{C^{2}+\alpha_{1}^{2}}{ }^{2}\right)\left(\beta_{1} x^{2}-\omega x+\sqrt{e_{C^{2}+\alpha_{1}^{2}}}\right)}\right.}{\beta_{1}{ }^{2}}\right\}
\end{aligned}
$$

\section{5. 計算結果と慣用計算値並びに実測した応力・タワミとの比較}

御秡橋については断面諸数值は次のと和りである。

$$
\begin{aligned}
& A_{1}=3000 \mathrm{~cm}^{2}, A_{2}=4000 \mathrm{~cm}^{2}, I_{1}=225000 \mathrm{~cm}^{4}, I_{2}=533000 \mathrm{~cm}^{4}, A_{S_{1}}=A_{S_{2}}=9.41 \mathrm{~cm}^{2}, \\
& E_{C}=3.5 \times 10^{5} \mathrm{~kg} / \mathrm{cm}^{2}, \quad E_{S}=2.0 \times 10^{6} \mathrm{~kg} / \mathrm{cm}^{2}, \quad e_{A}=12.5 \mathrm{~cm}, \quad e_{B}=9 \mathrm{~cm}, \quad e_{C}=11 \mathrm{~cm}, \\
& l_{1}=490 \mathrm{~cm}, \quad h=250 \mathrm{~cm}, \quad \xi=75 \mathrm{~cm}
\end{aligned}
$$

（a） 不静定力 不静定力は PC 鋼線の付加的な影響を考慮に入れない場合の普通のラーメンの計算，すなわ ら慣用計算法によつて計算すれば $H_{0}=0.893 \mathrm{~W}$ である。 これを本文計算法によれば $H=0.913 W$ となり，慣 用計算值にくらべ大きな值を与え， $H / H_{0}=1.023$ である。このととほ前述したごとく $\mathrm{PC}$ 鋼線の付加的な緊張力 または弛緩力による影響は不静定構造物では 不静定力に関係するので無視し得ないといろことを裏書きしている ものであり，これがため当然構造物各部の応力状態，タワミなどが変つてくるという結果になる。

(b) 曲げモーメント及び軸力 ハリ部及び脚柱部に捻ける曲げモーメントと軸力を列記すれば 表一1，2 とな

表一1 ハリ部における曲げモーメント及び軸力

$(M: W \mathrm{~kg}-\mathrm{cm} N: W \mathrm{~kg})$

\begin{tabular}{l|r|r|r|r|r|r|c}
\hline$x \mathrm{~cm}$ & \multicolumn{1}{|c|}{0} & 75.0 & 122.5 & 245.0 & 367.5 & 437.5 & 490.0 \\
\hline$M_{1}(x)$ & 184.98 & 185.40 & 139.60 & 19.94 & -102.44 & -171.53 & -220.88 \\
$M_{10}(x)$ & 191.77 & 191.77 & 144.27 & 22.77 & -100.73 & -170.73 & -223.23 \\
$N_{1}(x)$ & 1.375 & 1.355 & 1.217 & 0.925 & 0.903 & 1.096 & 1.364 \\
$N_{10}(x)$ & 0.893 & 0.893 & 0.893 & 0.893 & 0.893 & 0.893 & 0.893 \\
\hline
\end{tabular}

たたし $M_{10}(x), N_{10}(x)$ は慣用計算值を示す
表一2 脚柱部における曲げモーメント及び軸力

$(M: W \mathrm{~kg}-\mathrm{cm} N: W \mathrm{~kg})$

\begin{tabular}{l|r|r|r|r|c}
\hline$y \mathrm{~cm}$ & 0 & 62.5 & 125.0 & 187.5 & 250.0 \\
\hline$M_{2}(y)$ & -220.88 & -166.80 & -111.75 & -56.04 & 0 \\
$M_{20}(y)$ & -223.23 & -167.42 & -111.62 & -55.81 & 0 \\
$N_{2}(y)$ & 1.267 & 1.145 & 1.058 & 1.004 & 0.987 \\
$N_{20}(y)$ & 1.000 & 1.000 & 1.000 & 1.000 & 1.000 \\
\hline
\end{tabular}

ただし $M_{20}(y), N_{20}(y)$ は慣用計算值を示す 


\section{図一4 ハリ部下縁における応カ}

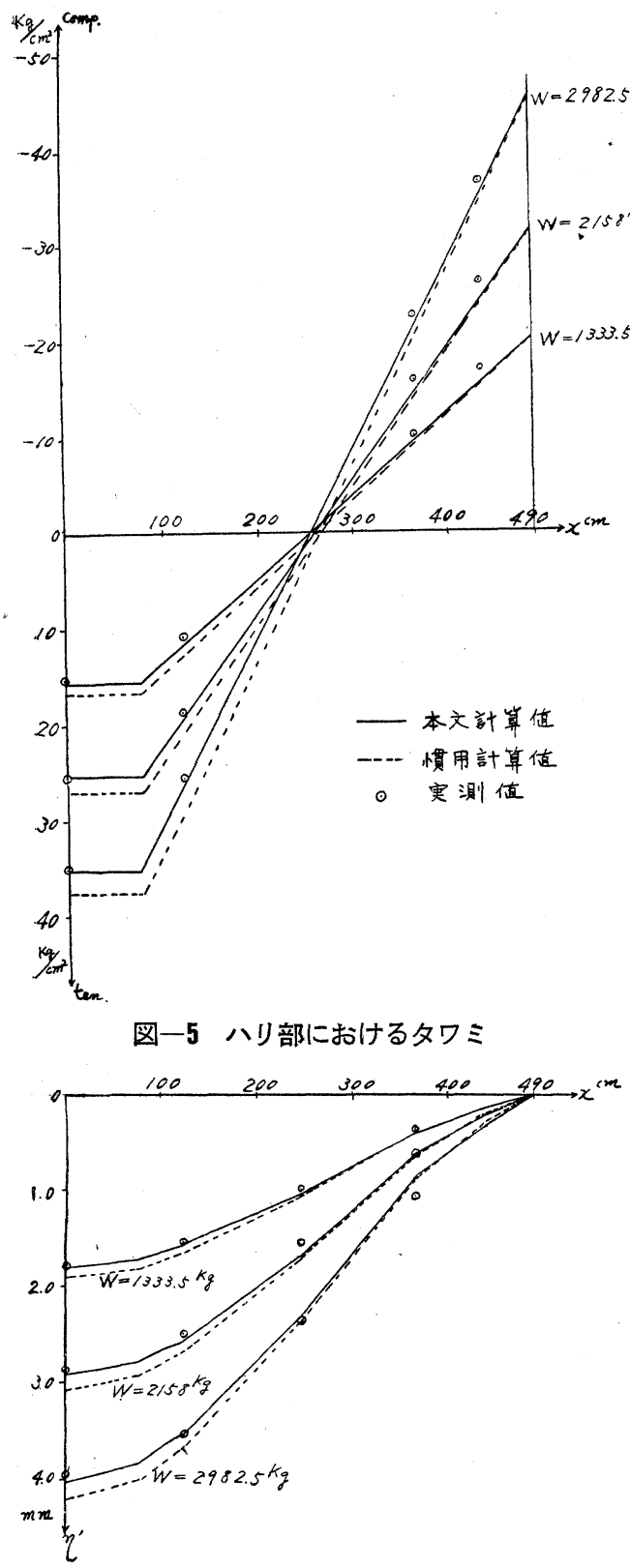

る。これらの表から明らかなどとく，ハリ中央 C 点汇 特ける本交計算法による曲げモーメントは慣用計算法 そよる值の $96 \%$ である。軸力は慣用計算法汒よれば ハリ部，脚柱部についてそれぞれ一定であるのと対し 本文計算法に上れば複雑沵経過を示している。

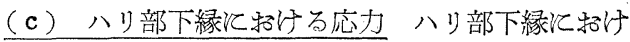
る実測値と計算値を比較したものが表一3でありこ れを図示したものが 図一4である。

これらの表や図から慣用計算値と実測值との間注 かなりの差が認められるが，本交計算法汇よれば大体 実測值と一致さすととができる。しかしながら 図一4 そ特いて実測値は計算值の上側にでてくる傾向を示し ている。すなわちハリ部に挌ける応力0の点がもつと 内側偏つた所淿ると云う事実を否定するととはで きない。これらの点とついては脚柱下端汸ける鉸の 固定度などが問題になつてくると思われる。

表一3 ハリ部下緣における応力 $\left(\mathrm{kg} / \mathrm{cm}^{2}\right)$

\begin{tabular}{|c|c|c|c|c|c|c|c|c|c|}
\hline \multirow{2}{*}{$\frac{\text { 位置 }}{x}$} & \multicolumn{3}{|c|}{$W=1333.5 \mathrm{~kg}$} & \multicolumn{3}{|c|}{$W=2158 \mathrm{~kg}$} & \multicolumn{3}{|c|}{$W=2982.5 \mathrm{~kg}$} \\
\hline & 実測値 & $\sigma$ & $\sigma_{0}$ & 実測値 & $\sigma$ & $\sigma_{0}$ & |実測值 & $\sigma$ & $\sigma_{0}$ \\
\hline 0 & 15.1 & 15.8 & 16.7 & 25.7 & 25.6 & 27.0 & 34.8 & 35.4 & 37.3 \\
\hline $75.0 \mathrm{~cm}$ & & 15.9 & 16.7 & & 25.7 & 27.0 & & 35.5 & 37.3 \\
\hline 122.5 & 10.5 & 11.9 & 12.4 & 18.7 & 19.2 & 20.1 & 25.2 & 26.6 & 27.8 \\
\hline 245.0 & 1.8 & 1.4 & 1.6 & 0.9 & 2.2 & 2.6 & 2.6 & 3.0 & 3.7 \\
\hline 367.5 & -10.5 & -9.5 & -9.4 & -16.1 & -15.4 & -15.1 & -23.4 & -21.3 & -20.9 \\
\hline 437.5 & $|-17.5|$ & -15.7 & -15.6 & -26.6 & -25.5 & -25.2 & -37.6 & -35.2 & -34.8 \\
\hline 490.0 & & -20.2 & -20.2 & & -32.8 & -32.8 & & -45.3 & -45.3 \\
\hline
\end{tabular}

ただし $\sigma \cdots \cdots$...本文計算值 $\quad \sigma_{0} \cdots \cdots \cdot$ 慣用計算值

表一4 ハリ部におけるタワミ

(mm)

\begin{tabular}{|c|c|c|c|c|c|c|c|c|c|}
\hline \multirow{2}{*}{$\frac{\text { 位置 }}{x}$} & \multicolumn{3}{|c|}{$W^{\prime}=1333.5 \mathrm{~kg}$} & \multicolumn{3}{|c|}{$W=2158 \mathrm{~kg}$} & \multicolumn{3}{|c|}{$W=2982.5 \mathrm{~kg}$} \\
\hline & 実測值 & $\eta^{\prime}(x)$ & $\eta_{0}^{\prime}(x)$ & 実測值 & $\eta^{\prime}(x)$ & $\eta_{0}^{\prime}(x)$ & 実測值 & $\eta^{\prime}(x)$ & $\eta_{0}^{\prime}(x)$ \\
\hline 0 & 1.80 & 1.82 & 1.88 & 2.87 & 2.94 & 3.05 & 3.94 & 4.07 & 4.21 \\
\hline $75.0 \mathrm{~cm}$ & & 1.93 & 1.99 & & 2.80 & 2.90 & & 3.87 & 4.00 \\
\hline 122.5 & 1.55 & 1.59 & 1.64 & 2.49 & 2.57 & 2.65 & 3.57 & 3.55 & 3.67 \\
\hline 245.0 & 0.98 & 1.04 & 1.05 & 1.54 & 1.68 & 1.69 & 2.34 & 2.32 & 2.34 \\
\hline 367.5 & 0.36 & 0.37 & 0.39 & 0.64 & 0.63 & 0.64 & 1.09 & 0.87 & 0.88 \\
\hline 440.0 & 0.13 & 0.13 & 0.12 & 0.20 & 0.20 & 0.19 & 0.28 & 0.28 & 0.26 \\
\hline 490.0 & & 0 & 0 & & 0 & 0 & 0 & 0 & 0 \\
\hline
\end{tabular}

たたしし $\eta^{\prime} \cdots . .$. 本交計算値 $\eta_{0}{ }^{\circ} \cdot \cdots \cdot$...慣用計算値

$\eta^{\prime}(x)$ は梁端部を 0 として下向きに正をとつた場合の挂みである i.e. $\eta^{\prime}(x)=\eta(490)-\eta(x)$

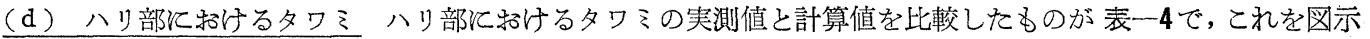
したるのが図一5である。実測值と慣用計算值との間には差が認められるが本文計算法によれば大体一致してくる。 乙かしながらこの場合にる実測值は計算值の上側にでてくる傾向を示し応力の場合の結果とよく符号している。

\section{6. 結 言}

以上飞 PC 鋼線の付加的な影響を考慮した場合のプレストレストコンクリート門構ラーメンの計算を示し，御

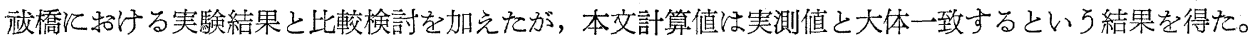

また部材の変形にとるなら PC 鋼線の付加的な影響は不静定構造物々招いては不静定力に関係するので考慮さ れて然るべきるのではなかららかと思われる。

最後飞実験飞当り御協力を弓けた七尾市当局，PS コンクリートK KV対し厚く感謝の意を表したい。

(昭.30.2.23) 